FOLIA

Amazónica

Revista del Instituto de Investigaciones

de la Amazonía Peruana

\title{
RELACIÓN LONGITUD PESO Y FACTOR DE CONDICIÓN DE Prochilodus nigricans Y Potamorhina altamazonica EN LA CUENCA DEL RÍO TAHUAYO, LORETO (PERÚ)
}

\author{
Carlos Martin CAÑAS ALVA ${ }^{1}$, Luis Carlos MOYA VÁSQUEZ2*, \\ Marina VARGAS ROJAS ${ }^{3}$, Armando MERCADO TORRES ${ }^{2}$ \\ 1 Investigador Independiente. Florida, USA. \\ 2 Wildlife Conservation Society, WCS. \\ 3 Instituto del Bien Común, IBC. \\ * Correo electrónico: Imoya@wcs.org
}

\section{RESUMEN}

Se presenta la relación Longitud-Peso y Factor de condición para Prochilodus nigricans (boquichico) y Potamorhina altamazonica (llambina) para la cuenca del río Tahuayo, dos especies de gran importancia comercial y para la pesca de subsistencia en muchos ríos y tributarios en Loreto, Perú. Durante 12 meses se colectaron datos biológicos de 1114 individuos de las dos especies a partir de las capturas realizadas por los pescadores de la Asociación de Pescadores Artesanales "Puma Garza I". Los resultados evidenciaron que boquichico tiene un crecimiento isométrico ( $\mathrm{PT}=0,0126 \times \mathrm{LT}^{\wedge 3.034}$ ) y llambina presenta un crecimiento alométrico negativo con diferencias en los parámetros para hembras $\left(\mathrm{PT}=0,0985 \times \mathrm{LT}^{\wedge 2.35}\right)$ y para machos $\left(\mathrm{PT}=0,0755 \times \mathrm{LT}^{\wedge 2.43}\right)$. Ambas especies presentaron un factor de condición relativo bueno durante todo el ciclo hidrológico y sus variaciones estuvieron relacionadas a las condiciones hidrológicas dela cuenca. Estas estimaciones proveen las primerasinformaciones del crecimiento de estas dos especies en función de la talla y el peso y son de gran utilidad para el conocimiento de los stocks pesqueros de estas especies distribuidas en la cuenca del río Tahuayo.

PALABRAS CLAVE: crecimiento isométrico, crecimiento alométrico, boquichico, llambina, Amazonía. 


\title{
LENGTH-WEIGHT RELATIONSHIP AND CONDITION FACTOR OF Prochilodus nigricans AND Potamorhina altamazonica IN THE TAHUAYO RIVER BASIN, LORETO (PERU)
}

\begin{abstract}
In this study, we present the Length-Weight relationship and Condition Factor for Prochilodus nigricans (boquichico) and Potamorhina altamazonica (llambina) from the Tahuayo River basin. These two species are among the most important commercial and subsistence fishing resources for Loreto, Peru. Biological data were collected during 12 months and analyses were obtained after 1114 individual records of these two species from catches provided by the Association of Artisanal Fishermen "Puma Garza I". Results showed that boquichico, both males and females, features an isometric growth $\left(\mathrm{PT}=0.0126 \times \mathrm{LT}^{3.034}\right)$ and llambina has a negative allometric growth with differences in the parameters for females $\left(\mathrm{PT}=0.0985 \times \mathrm{LT}^{2.35}\right)$ and for males $\left(\mathrm{PT}=0.0755 \times \mathrm{LT}^{2.43}\right)$. Fish stocks of both species were found in good fitness condition throughout the hydrological cycle and variations were related to the hydrological conditions of the basin. These estimates provide the first information about growth of these two species as a function of size and weight and will be very useful in the management of their fish stocks distributed in the Tahuayo River basin.
\end{abstract}

KEYWORDS: isometric growth, allometric growth, boquichico, llambina, Amazon basin. 


\section{INTRODUCCIÓN}

El conocimiento de las características morfométricas de los recursos pesqueros constituye la base para la investigación de las pesquerías, su utilidadabarca desdeladiferenciacióntaxonómica hasta el establecimiento de acciones de manejo sobre determinado recurso en explotación (Schneider et al., 2000; Tobes et al., 2016; Froese et al., 2011). Dentro de estos parámetros, la relación Longitud-Peso es clave para entender el patrón de crecimiento del recurso y estimar valores de biomasa a partir de grupos de talla, o incluso, para identificar variaciones del estado físico y salud de las poblaciones, muchas veces asociada a la calidad y estado de conservación de los hábitats acuáticos (Le Cren, 1951; Froese \& Pauly, 2000; Schneider et al., 2000; Froese et al., 2011). Asimismo, esta relación es de utilidad en la comparación de las distintas poblaciones de una misma especie de amplia distribución (Tobes et al., 2016) y está siendo considerada por varios autores como una herramienta para la evaluación y monitoreo de potenciales impactos ambientales en ecosistemas acuáticos (Giarrizzo et al., 2011; Chuctaya et al., 2017). En el caso de los recursos pesqueros amazónicos, generar este conocimiento toma mayor relevancia sobre todo para aquellas especies migratorias, pues en ese permanente tránsito, estas especies hacen uso de distintos hábitats para cumplir funciones vitales asociadas a las condiciones hidrológicas de los ecosistemas (Goulding et al., 2018). En la mayoría de casos, algunas poblaciones, o parte de ellas, ocupan determinados hábitats, cada uno con condiciones abióticas y bióticas que influyen en el patrón de crecimiento y el estado físico de las especies (Araujo-Lima \& Ruffino, 2003; Froese, 2006; Mensah, 2015). El factor de condición (K) nos indica estado de salud, bienestar o la condición fisiológica de los individuos de una población y su estimación está basada en la relación del peso adquirido por un pez con respecto a determinada talla, bajo el supuesto, a mayor peso en cierto rango de talla, mejor es el factor de condición (Froese, 2006). Es importante destacar, sin embargo, que este índice del estado de salud de un pez no solo depende del supuesto que el peso de un pez es proporcional al cubo de su longitud (Ecuación de Fulton: $\mathrm{K}=\mathrm{PT} / \mathrm{LT}^{3}$ ), sino también va a estar influenciado por factores bióticos (edad, sexo, grupo taxonómico) y abióticos (estación hidrológica y disponibilidad de alimento) de los lugares donde se distribuyan sus poblaciones (Le Cren, 1951; Froese, 2006). Es decir, existe una variabilidad natural del factor de condición de un pez que está en función de su patrón real de crecimiento, índice conocido como Factor de Condición Relativo ( $\mathrm{K}_{\mathrm{rel}}$ ) (Le Cren, 1951), que es el más recomendado utilizar para identificar la condición o forma de los individuos con el incremento de su tamaño.

En el Perú, la producción de estudios sobre la relación Longitud-Peso para peces amazónicos es todavía limitada. La literatura existente muestra resultados solo para 56 especies, de un total de 1 141 especies reportadas para aguas continentales peruanas (MINAM, 2019). Los estudios han sido obtenidos a partir de datos colectados en estanques piscícolas (Felipa et al., 2016), a partir de desembarques en principales centros urbanos (Riofrío, 2002; García et al., 2010; Flores-Gómez, 2015; Riofrío-Quijandría et al., 2017; BonillaCastillo et al., 2018), y en algunos casos para determinadas cuencas (Alcántara et al., 2008; Tobes et al., 2015; Chuctaya et al., 2017; GarcíaAyala, 2017). Prochilodus nigricans (Familia Prochilodontidae) y Potamorhina altamazonica (Familia Curimatidae) son dos especies de gran importancia en la economía regional de Loreto. Por varias décadas han sido las especies más explotadas comercialmente (Tello \& Bayley, 2001; 
García et al., 2009; García-Dávila et al., 2018). Ecológicamente, desempeñan un rol fundamental en el ciclo de la materia orgánica de los extensos bosques inundables amazónicos (Tello \& Bayley, 2001; Castro \& Vari, 2003; García et al., 2009; Goulding et al., 2018). Estas especies también destacan por su importante rol en la pesca de subsistencia y en la economía a pequeña escala de las comunidades ribereñas establecidas en los bosques inundables de la Amazonía loretana (Anderson et al., 2009; Padoch \& Steward, 2010), y la cuenca del río Tahuayo no es la excepción. Desde hace varias décadas las comunidades establecidas a las orillas del río Tahuayo explotan estas especies, lo cual les ha permitido hacer un comercio a pequeña escala y ha sido de gran importancia para su sustento familiar y para la organización comunal (Smith et al., 2001; Chirif, 2012).

El presente estudio tiene por objetivo analizar y presentar la relación Longitud-Peso y evaluar el Factor de Condición de Prochilodus nigricans y Potamorhina altamazonica en la cuenca del río Tahuayo. Esta información va a permitir identificar el patrón de crecimiento y estado de salud actual de estas dos especies en la cuenca, como punto de partida para proponer futuras evaluaciones biológicas y pesqueras de sus poblaciones. Este estudio también va a poner a disponibilidad los datos morfométricos de estas dos especies a la base de datos FishBase (Froese \& Pauly, 2019), la cual actualmente no incluye información de Perú.

\section{MATERIAL Y MÉTODOS}

\section{ÁREA DE ESTUDIO}

El río Tahuayo es un tributario de aguas negras ubicado en la margen derecha del río Amazonas a $65 \mathrm{~km}$ al sur de la ciudad de Iquitos, principal centro comercial en la región Loreto (Perú).
Comprende un área de drenaje de $1400 \mathrm{~km}^{2}$, que se caracteriza por la presencia de un bosque de colinas bajas en su lado este (asociadas al río Blanco, su único tributario) y por un extenso bosque aluvial inundable y pantano de palmeras asociados a un sistema de tahuampas, restingas y lagunas (cochas) en el lado oeste (Figura 1). Durantela época de lluvias el río Amazonas inunda el lado oeste de la cuenca e incrementa el nivel de agua entre 6-8 metros, dejando sumergidos extensos bosques y permitiendo el ingreso de peces a todo el sistema de cochas (Coomes, 1995). El régimen hidrológico de la cuenca es controlado por el río Amazonas y no existe registro histórico de mediciones de los niveles del río Tahuayo. Tomando como base la identificación de meses con mínimos caudales en la estación Tamshiyacu (Espinoza et al., 2009) y los registros mensuales de mediciones de nivel de agua reportados para la estación Iquitos por el Servicio de Hidrografía y Navegación de la Amazonía (Marina de Guerra del Perú, 2018), se asumen cuatro estaciones hidrológicas para la cuenca para fines de análisis: época de vaciante (agosto, septiembre y octubre), época de transición a creciente (noviembre, diciembre y enero), época de creciente (febrero, marzo y abril) y época de transición a vaciante (mayo, junio y julio).

\section{MUESTREO BIOLÓGICO}

El muestreo biológico se llevó a cabo durante el periodo hidrológico 2016-2017, entre los meses de agosto (vaciante) y julio (transición a vaciante), en la cuenca del río Tahuayo. El registro de las capturas se realizó en las comunidades El Chino, Buena Vista y San Pedro, en horarios de 6:00 a 9:30 am y 3:30 a 5:30 pm, y con la participación de miembros con la Asociación de Pescadores Artesanales "Puma Garza I" de dichas comunidades. Los aparejos de pesca utilizados fueron flechas y redes de tipo trampera con diferentes tamaños de abertura de malla: 1,5 x 3 


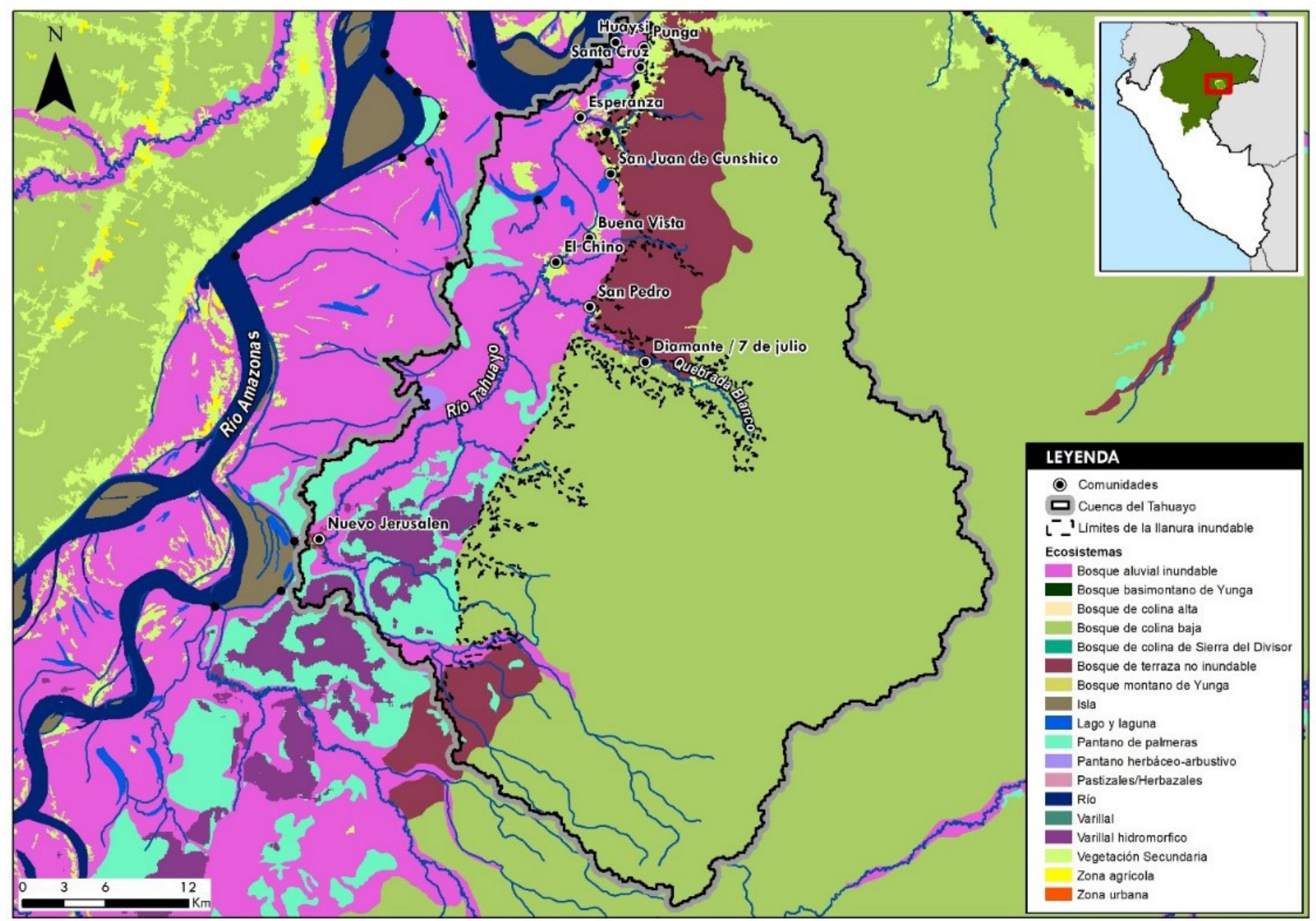

Figura 1. La cuenca del río Tahuayo y sus ecosistemas acuáticos relacionados al río Amazonas, en Loreto (Perú).

pulgadas, $2,5 \times 3$ pulgadas, $3 \times 3$ pulgadas y $4 \times 3$ pulgadas cada uno de $1 / 2$ paño de longitud. Para determinar la longitud de los individuos se utilizó un ictiómetro de metal con unidades mínimas de $1 \mathrm{~mm}$ y se utilizó la longitud total del pez (LT), que comprende desde el extremo más anterior del cuerpo hasta el borde posterior de la aleta caudal. El peso fue determinado con el uso de una balanza digital con sensibilidad 0,1 gr.

\section{Análisis de datos}

Cada uno de los ejemplares de las dos especies representó un registro en nuestra base de datos que incluyó las siguientes variables: fecha y procedencia (Zona de Pesca) de la captura, código del individuo, nombre de la especie, longitud y peso total, sexo y características de las gónadas. La relación Longitud-Peso fue evaluada tomando como punto de partida la relación $\mathrm{PT}=\mathrm{aLT}^{\mathrm{b}}$, donde $\mathrm{PT}$ es el peso total en gramos, $\mathrm{LT}$ es la longitud total en centímetros, $a$ (intercepto) y $b$ (pendiente) son los coeficientes que predicen el patrón de crecimiento en cada una de las especies en función del tamaño y el peso. Los valores de estos dos coeficientes fueron obtenidos por el método Tipo I o predictivo (Ricker, 1975; Froese \& Pauly, 2000) luego de transformar la relación exponencial de la longitud y peso en una relación lineal con la aplicación del logaritmo natural: $\mathrm{Ln}(\mathrm{PT})=a+b \operatorname{Ln}(\mathrm{LT})$. La condición somática 
Tabla 1: Parámetros de la relación Longitud-Peso, Factor de condición somática y principales aspectos biométricos estimados para Prochilodus nigricans (boquichico) y Potamorhina altamazonica (llambina) en la cuenca del Tahuayo, Loreto (Perú).

\begin{tabular}{|c|c|c|c|c|c|c|}
\hline \multirow[b]{2}{*}{ Parámetro } & \multicolumn{3}{|c|}{ Prochilodus nigricans } & \multicolumn{3}{|c|}{ Potamorhina altamazonica } \\
\hline & $\begin{array}{c}\text { Ambos } \\
\text { sexos }\end{array}$ & Hembra & Macho & $\begin{array}{c}\text { Ambos } \\
\text { sexos }\end{array}$ & Hembra & Macho \\
\hline a & 0,01263 & 0,01313 & 0,01285 & 0,08427 & 0,09857 & 0,07549 \\
\hline$b$ & 3,034 & 3,026 & 3,025 & 2,4 & 2,352 & 2,432 \\
\hline$r^{2}$ & 0,95 & 0,94 & 0,96 & 0,86 & 0,83 & 0,89 \\
\hline $\mathrm{N}$ & 540 & 278 & 262 & 574 & 316 & 259 \\
\hline Longitud Total (LT) promedio & 22,81 & 23,02 & 22,58 & 21,76 & 21,89 & 21,59 \\
\hline LT máxima & 37,5 & 37,5 & 30,7 & 27,9 & 27,9 & 26,7 \\
\hline LT mínima & 12 & 12 & 12 & 13,6 & 17,8 & 13,6 \\
\hline Intervalo Confianza (LT) & 0,283 & 0,386 & 0,416 & 0,154 & 0,203 & 0,236 \\
\hline Peso Total (PT) promedio & 178,11 & 184,8 & 171,02 & 138,9757 & 142,39 & 139,93 \\
\hline PT máximo & 685 & 685 & 390 & 278 & 278 & 226 \\
\hline PT mínimo & 22 & 22 & 23 & 30 & 94 & 30 \\
\hline Intervalo Confianza 95\% (PT) & 6,518 & 9,516 & 8,844 & 2,645 & 3,734 & 3,678 \\
\hline $\mathrm{K}_{\text {rel }}$ promedio & 1,006 & 1,018 & 0,993 & 1,004 & 1,004 & 1,003 \\
\hline $\mathrm{K}_{\mathrm{rel}}$ máximo & 1,371 & 1,371 & 1,325 & 1,323 & 1,323 & 1,228 \\
\hline $\mathrm{K}_{\mathrm{rel}}$ mínimo & 0,755 & 0,755 & 0,787 & 0,648 & 0,791 & 0,648 \\
\hline Intervalo Confianza 95\% $\left(\mathrm{K}_{\mathrm{rel}}\right)$ & 0,009 & 0,014 & 0,013 & 0,007 & 0,009 & 0,009 \\
\hline
\end{tabular}

fue evaluada usando el Factor de Condición Relativo $\left(\mathrm{K}_{\mathrm{rel}}\right)$ presentado por Le Cren (1951) y recomendado por Froese (2006): $\mathrm{K}_{\mathrm{rel}}=\mathrm{PT} / a \mathrm{~L} b$. El valor $b$ calculado en la relación longitud-peso para cada una de las especies fue utilizado para estimar el $\mathrm{K}_{\text {rel }}$.

Todos los análisis de significancia estadística consideraron un $95 \%$ de confiabilidad $(p$-valor $=$ $0,05)$. Se utilizó análisis de chi-cuadrado $\left(\mathrm{X}^{2}\right)$ para comparar la proporción de sexos (asumiendo la relación 1:1) y el análisis de Varianza de un factor (ANOVA), que permite determinar si existen diferencias significativas entre las medias de múltiples muestras tomando como variables independientes los periodos hidrológicos y sexo. Todos los cálculos se realizaron utilizando MS Excel.

\section{RESULTADOS}

Entre agosto 2016 y abril 2017 se evaluaron un total de 1114 individuos de las dos especies en estudio, 540 fueron boquichicos y 574 llambinas. Los parámetros $a$ y $b$ de la relación LongitudPeso y Factor de Condición relativo $\left(\mathrm{K}_{\mathrm{rel}}\right)$ estimados, así como el tamaño de la muestra, proporción de sexos, rango de tallas y pesos, sus 
Tabla 2: Diferencias entre las varianzas de los residuos resultantes de la linealización de longitud y peso.

\begin{tabular}{lcccccc}
\hline $\begin{array}{c}\text { Origen de las } \\
\text { variaciones }\end{array}$ & $\begin{array}{c}\text { Suma de } \\
\text { cuadrados }\end{array}$ & $\begin{array}{c}\text { Grados de } \\
\text { Libertad }\end{array}$ & $\begin{array}{c}\text { Promedio de los } \\
\text { Cuadrados }\end{array}$ & F & p & $\begin{array}{c}\text { Valor F } \\
\text { crítico }\end{array}$ \\
\hline Entre grupos & $1,77636 \mathrm{E}-15$ & 1 & $1,77636 \mathrm{E}-15$ & $<0,001$ & 0,9999 & 3,8588 \\
Dentro de los grupos & 6,362660414 & 538 & 0,011826506 & & & \\
Total & 6,362660414 & 539 & & & & \\
\hline
\end{tabular}

Tabla 3: Análisis de varianzas entre $\mathrm{K}_{\text {rel }}$ por periodos hidrológicos.

\begin{tabular}{lcccccc}
\hline $\begin{array}{c}\text { Origen de las } \\
\text { variaciones }\end{array}$ & $\begin{array}{c}\text { Suma de } \\
\text { cuadrados }\end{array}$ & $\begin{array}{c}\text { Grados de } \\
\text { Libertad }\end{array}$ & $\begin{array}{c}\text { Promedio de los } \\
\text { Cuadrados }\end{array}$ & $F$ & $p$ & $\begin{array}{c}\text { Valor F } \\
\text { crítico }\end{array}$ \\
\hline Entre grupos & 1,058886485 & 3 & 0,352962162 & 33,833 & $<0,001$ & 2,6218 \\
Dentro de los grupos & 5,518838457 & 529 & 0,010432587 & & & \\
Total & 6,577724942 & 532 & & & \\
\hline
\end{tabular}

respectivos valores de factor de determinación $\left(r^{2}\right)$ e intervalos de confianza (95\%) se muestran en la Tabla 1. Al aplicar la prueba de chi-cuadrado $\left(X^{2}\right)$ a la proporción entre hembras y machos de boquichico, no se encontraron diferencias significativas, $X^{2}(1, N=540)=0,4758, p=0,49, \mathrm{y}$ en el caso de llambina la proporción de hembras fue significativamente mayor a la proporción de machos, $X^{2}(1, N=575)=5,65, p=0,017$.

La relación longitud peso para boquichico (Prochilodusnigricans) enlacuencadelrío Tahuayo resultó en $\mathrm{PT}=0,0126(\mathrm{LT})^{3.034}$ y los coeficientes $a \mathrm{y}$ $b$ obtenidos para todos los individuos obtuvieron un coeficiente de determinación $r^{2}=0,95$. Los coeficientes $a$ y $b$ obtenidos para hembras y machos resultaron diferentes, sin embargo, no se encontraron diferencias significativas $(\mathrm{F}<0$; $p>$ $0,05)$ al momento de analizar las varianzas de los residuos de sus respectivas regresiones lineales (Tabla 2), por lo que se propone usar una sola ecuación de crecimiento para esta especie en la cuenca Tahuayo.

El factor de condición relativo $\left(\mathrm{K}_{\mathrm{rel}}\right)$ de boquichico fluctuó entre 0,76 y 1,37: Las hembras siempre mostraron mayores valores de condición somática que los machos, lo cual fue evaluado estadísticamente con $95 \%$ de confiabilidad $(\mathrm{F}=6,88 ; p=0,009)$. Al comparar el promedio de estos valores por periodos hidrológicos (Figura 2 ), se encontraron diferencias significativas ( $\mathrm{F}=$ 33,8305; $p<0,0001$ ) con un $95 \%$ de confiabilidad $(\mathrm{p}<0,05)$ entre cada uno de los periodos hidrológicos, identificándose un ascenso en $\mathrm{K}_{\text {rel }}$ desde la época de vaciante $(0,95)$ hasta el inicio de la transición a vaciante $(1,06)$ (Tabla 3$)$.

La relación Longitud-Peso para Potamorhina altamazonica (llambina) resultó determinada por PT $=0,0843$ (LT) ${ }^{2.4}$ para ambos sexos, con un coeficiente de determinación $r^{2}=0,86$. En este caso, los coeficientes obtenidos para hembras ( $a=$ 0,0985 y $\left.b=2,35 ; r^{2}=0,83\right)$ y machos $(a=0,0755$ y $\left.b=2,43 ; r^{2}=0,89\right)$ resultaron estadísticamente diferentes por lo cual se propone una relación longitud-peso para cada sexo: $\mathrm{PT}=0,0985(\mathrm{LT})^{2.35}$ para las hembras y PT $=0,0755(\mathrm{LT})^{2.43}$ para los machos. El factor de condición relativo $\left(\mathrm{K}_{\text {rel }}\right)$ fluctuó entre 0,65 y 1,32, y no se encontraron diferencias significativas $(F=0,019 ; p=0,89$ ) 


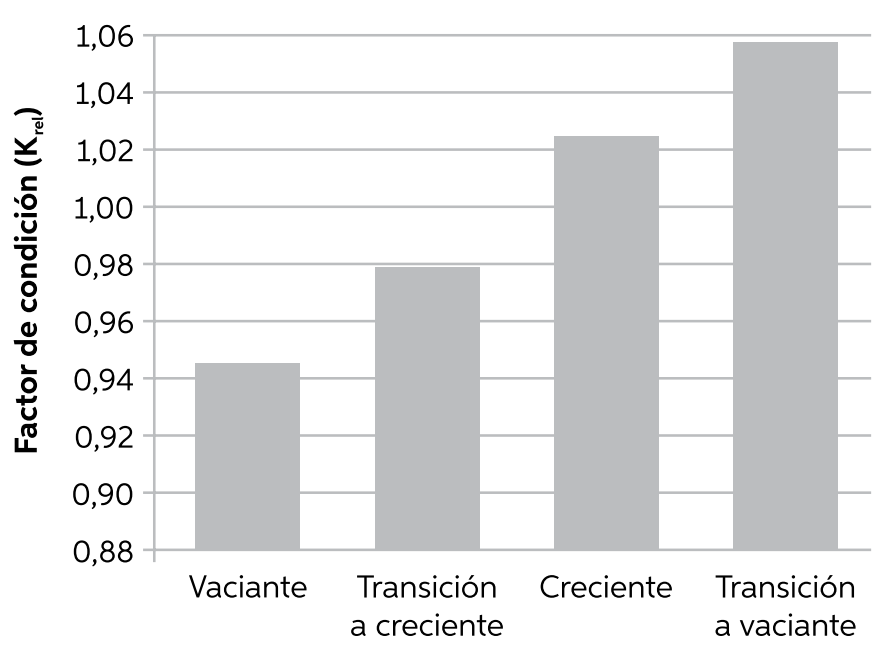

Figura 2: Factor de condición $\left(K_{\text {rel }}\right)$ promedio para Prochilodus nigricans por ciclo hidrológico en la cuenca del río Tahuayo, Loreto (Perú).

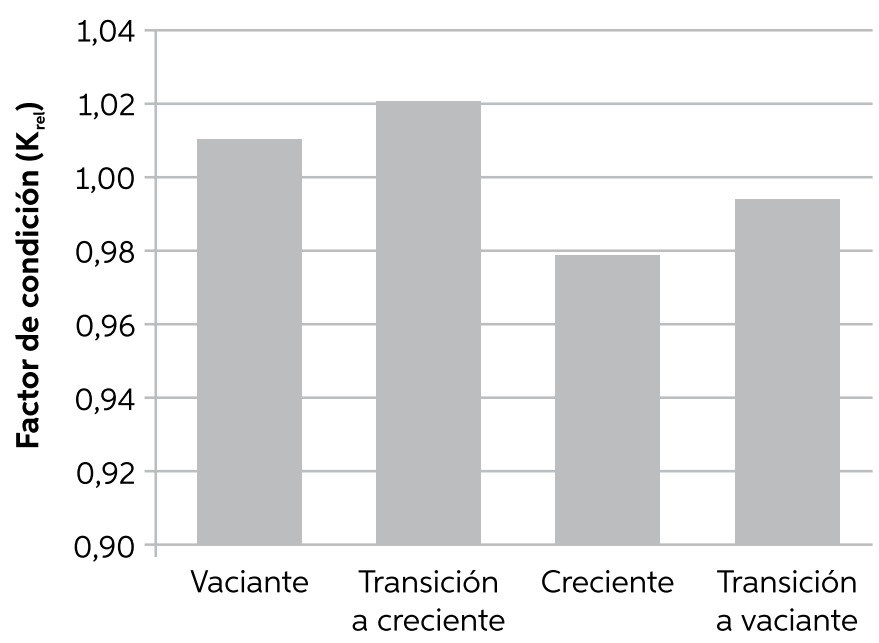

Figura 3: Factor de condición $\left(\mathrm{K}_{\mathrm{rel}}\right)$ promedio para Potamorhina altamazonica por ciclo hidrológico en la cuenca del río Tahuayo, Loreto (Perú).

con $95 \%$ de confiabilidad entre los valores de $\mathrm{K}_{\text {rel }}$ de hembras y machos durante el periodo de muestreo. Al observar los valores por periodo hidrológico, los valores de $\mathrm{K}_{\text {rel }}$ resultaron estadísticamente diferentes $(\mathrm{F}=9,10 ; p<0,0001)$ con los mayores valores en la primera etapa del ciclo hidrológico evaluado: entre la vaciante y el inicio de la creciente y menores valores durante la creciente (Figura 3).

\section{DISCUSIÓN}

El conocimiento de la relación longitud-peso es importante para describir aspectos de la biología de las especies, incrementa el conocimiento de los stocks pesqueros y es usado para entender el patrón de crecimiento de sus poblaciones (Froese \& Pauly, 2000; Giarrizo et al., 2011; DiebMagalhaes, 2015). En recursos pesqueros, la talla es biológicamente más importante que la edad, muchas de las funciones ecológicas (por ejemplo, el primer desove) están asociadas más a la talla del individuo que a la edad (Ricker, 1975). Conocer estas regresiones permite la conversión de la longitud de un individuo a peso o viceversa, o comparar los parámetros estimados de la ecuación de crecimiento entre diferentes años para identificar la condición o robustez de la población en el tiempo (Froese \& Pauly, 2000; De la Hoz et al., 2015). En la actualidad, se conoce la relación longitud-peso para Prochilodus nigricans y Potamorhina altamazonica a partir de muestreos de los desembarques de flotas comerciales en los ríos Putumayo, Amazonas y Ucayali (Riofrío, 2002; García et al., 2010; Flores-Gómez, 2015; Bonilla-Castillo et al., 2018). Los resultados obtenidos en este estudio describen por primera vez estas relaciones con información procedente de la cuenca del río Tahuayo y deberían ser considerados dentro futuros estudios de biología pesquera dentro de la cuenca y regulación pesquera.

La relación encontrada entre las variables longitud y peso es explicada con mayor exactitud para Prochilodus nigricans $\left(\mathrm{r}^{2}=0 ; 93\right)$ y describe de manera satisfactoria para el caso de Potamorhina altamazonica $\left(\mathrm{r}^{2}=0,86\right)$. El coeficiente $b$ obtenido para Prochilodus nigricans no fue estadísticamente diferente de 3 (F = $13,95, p<0,01$ ), es decir, esta especie presenta un crecimiento isométrico, los individuos mantienen un incremento proporcional de las 
dimensiones del cuerpo (peso y talla) durante todo su crecimiento (Ricker, 1975; Froese et al., 2011). El coeficiente $b$ para machos y hembras de Potamorhina altamazonica fue menor a 3, por lo que se determina que esta especie tiene un crecimiento hipoalométrico, es decir, el peso del cuerpo se incrementa a un ritmo menor que la talla del individuo, el pez crece más en longitud que en peso (Froese, 2006). Los parámetros obtenidos $(a \mathrm{y} b$ ) para ambas especies resultaron diferentes de valores obtenidos en previos estudios realizados tanto en Brasil como en Perú. Prochilodus nigricans reportó valores $\mathrm{a}=0,0095$ y $\mathrm{b}=3,1785$ para la zona de Santarém (Ruffino \& Isaac, 1995), para el lago Manacapurú (río Solimões) $\mathrm{a}=0,0636$ y $\mathrm{b}=2,72$ (Catarino et al., 2014) y para el río Madeira a=0,02972 y $\mathrm{b}=2,933$ (Cella-Ribeiro et al., 2015). Potamorhina altamazonica reportó valores de a=0.0211 y $\mathrm{b}=2.8592$ para el río Ucayali (Riofrio, 2002) y para el río Madeira $a=0,03409$ y b=2,869 (Cella-Ribero et al., 2015). Cuando se comparan los coeficientes estimados de las relaciones longitud-peso con las existentes en la literatura para una misma especie, siempre ocurrirá una amplia variabilidad debido a que estas relaciones de parámetros biológicos están fuertemente afectadas por variabilidad en las poblaciones y por los factores ambientales (Frota et al., 2004). Esta variabilidad encontrada también se debe atribuir a una combinación de factores como número de especímenes examinados, efectos de estacionalidad, la variabilidad y calidad de hábitat (Sarkar et al., 2013). En un meta-análisis de la relación longitud-peso en más de 2000 especies de peces en el mundo, se identificó que el valor del coeficiente $b$ regularmente varía dentro del rango 2,5-3,5 (Froese, 2006), rango al que corresponden los resultados obtenidos para Prochilodus nigricans. Los valores de $b$ obtenidos para Potamorhina altamazonica no se encuentran dentro de este intervalo. Varios autores mencionan que los valores de $b<2,5$ usualmente provienen de estudios que incluyeron una muestra muy pequeña de datos (Froese, 2006; Frota et al., 2004). La relación longitud-peso obtenida en este estudio para llambina proviene de 575 individuos evaluados, supera largamente los 100 individuos recomendados en este tipo de estudios (Froese et al., 2011) y se descarta la selectividad de los aparejos, dado que se usaron diferentes aparejos y tamaños de malla.

La relación longitud-peso puede ser usada para estimar el factor de condición de las especies y refleja las condiciones fisiológicas de alimentación de los individuos (Le Cren, 1951). Ambas especies presentaron un factor de condición $\left(\mathrm{K}_{\mathrm{rel}}\right)$ promedio alrededor de la unidad (Tabla 1), lo cual indica su buen estado de salud y las condiciones ambientales favorables de la cuenca. Ambas especies tienen un comportamiento altamente migratorio (Castro \& Vari, 2003; Goulding, 2018; Prieto, 2018) y sus stocks pesqueros están muy asociados a buscar alimento y refugio en el bosque inundable (Goulding, 1980; Saint-Paul et al., 2000). Disponibilidad de alimento o buena calidad de agua, son factores ambientales que otros autores han identificado como determinantes en el factor de condición de las especies (Olaya-Nieto, 2008; Zargar, 2012; Famoofo et al., 2020). Dado que el factor de condición relativo utilizado en estos análisis minimiza el efecto de eventos de madurez gonadal y reproducción, presencia o ausencia de parásitos o efectos de llenura del estómago (Morato et al., 2001; Lizama et al., 2006; Hanjavanit et al., 2013), la variación de los valores de $\mathrm{K}_{\text {rel }}$ obtenidos están reflejando principalmente cambios en las condiciones ambientales, como la presencia y acceso a zonas de alimentación. Las condiciones ambientales y productividad de la cuenca del río Tahuayo están asociadas al pulso de inundación del río Amazonas, que durante los meses de transición a creciente y creciente, 
permiten un incremento y acúmulo de materia orgánica disponible (detrito), lo cual representa una mayor oferta de alimento para especies detritívoras, como boquichico y llambina, favoreciendo así sus condiciones de salud dentro de la cuenca (Goulding, 1980; Junk et al., 1989; Mortillaro, 2011; Caraballo et al., 2012). El detrito se acumula y deposita tanto en el fondo de los cuerpos de agua como sobre toda la vegetación sumergida (hojas, ramas), lugares donde frecuentemente se alimentan estas especies (Yossa \& Araujo-Lima, 1998; observaciones de campo). Al evaluar los valores promedio de $\mathrm{K}_{\text {rel }}$ por cada uno de los periodos hidrológicos Prochilodus nigricans presentó menores valores para la vaciante (agosto 2016) y mantuvo un incremento hasta el final de la creciente (abril 2017), lo cual indicaría que a medida que se establece el periodo de máxima inundación mejoran las condiciones ambientales para esta especie, específicamente en la disponibilidad de alimento. Los valores de $\mathrm{K}_{\text {rel }}$ para Potamorhina altamazonia resultaron con los menores valores para la época de creciente, resultados similares a los obtenidos en estudios anteriores, García et al. (2010) reportaron que esta especie presenta los menores valores para los meses de diciembre a febrero y coinciden con la época de reproducción, temporada que dejan de alimentarse y utilizan todas sus reservas para el momento reproductivo. En la región Ucayali, los mayores valores de $\mathrm{K}$ para esta misma especie fueron encontrados para la época de vaciante y fueron asociados a la presencia de una mejor calidad de detrito en la época seca (Flores-Gómez, 2015).

\section{CONCLUSIONES}

Este estudio provee el primer análisis de la relación Longitud-Peso para dos especies de importancia en el consumo humano que habitan la cuenca del río Tahuayo y evalúa parámetros morfométricos de especies de la pesca comercial de Loreto luego de casi una década. Los índices de buen estado de salud obtenidos para estas dos especies sugieren implicancias favorables sobre los stocks pesqueros que habitan la cuenca del río Tahuayo. Los coeficientes obtenidos de la relación Longitud-Peso sugieren que los stocks pesqueros de boquichico y llambina tienen un patrón de crecimiento diferente a los observados en otras cuencas, y dada su importancia comercial para la región Loreto y, en particular, para la subsistencia de las poblaciones asentadas en el río Tahuayo, las acciones de manejo que se propongan sobre estas dos especies, como por ejemplo talla mínima de captura o aparejos permitidos (tamaños de malla), deben considerar estos resultados tanto al interior de la cuenca (acciones de control $\mathrm{y}$ vigilancia) como a nivel de las regulaciones regionales de pesca. Asimismo, estas diferencias obtenidas deben tomarse como base para entender la importancia de pequeñas cuencas del bosque inundable en el mantenimiento de los stocks pesqueros en la Amazonía peruana. Se deben promover más estudios sobre relaciones longitud peso y factor de condición para una mejor comprensión de la variabilidad de estos parámetros en la región (por cuencas, por ejemplo) o para otras especies dentro de la cuenca del río Tahuayo y tener mayor conocimiento del crecimiento de sus poblaciones. Adicionalmente, se recomienda que los pescadores ribereños tomen una participación más activa en la generación y difusión de este conocimiento, a fin de evitar la falta a las regulaciones de pesca y hacer más eficiente el manejo pesquero.

\section{AGRADECIMIENTOS}

Agradecemos de manera especial a los pescadores de las comunidades Buena Vista, El 
Chino y San Pedro, a la directiva de la Asociación de Pescadores Artesanales "Puma Garza I", y al Comité de Gestión del Área de Conservación Regional Tamshiyacu Tahuayo (ACR CTT), por el apoyo y facilidades brindadas durante las actividades de monitoreo de la pesca comunitaria. A Mariana Montoya, Sandra Ríos y José Mena por los importantes aportes realizados a la versión preliminar del manuscrito. Finalmente, este trabajo ha sido posible gracias al apoyo financiero de la Fundación Gordon \& Betty Moore y The Arcadia Charitable Trust.

\section{REFERENCIAS BIBLIOGRÁFICAS}

Alcántara, F.; Chu-Koo, F.; Rodríguez, L.; Chávez, C.; Tello, S.; Nuñez, J. 2008. Relación longitudpeso y el factor de condición del zúngaro tigrinus Brachyplatystoma tigrinum del río Amazonas, Loreto, Perú. Folia Amazónica,17(12): 23-28. DOI: https://doi.org/10.24841/ fa.v17i1-2.263

Anderson, E.; Montoya, M.; Soto, A.; Flores, H. 2009. Challenges and opportunities for comanagement of a migratory fish (Prochilodus nigricans) in the Peruvian Amazon. In: Haro, A.J.; Smith, K.L.; Rulifson, R.A.; Moffitt, C.M.; Klauda, R.J.; Dadswell, M.J.; Cunjak, R.A.; Cooper, J.E.; Beal, K.L.; Avery, T.S. (Eds). Challenges for diadromous fishes in a dynamic global environment. Bethesda, MD: American Fisheries Society, Symposium 69. p 1-16.

Araujo-Lima, C.A.R.M.; Ruffino, M.L. 2003. Migratory fishes of the Brazilian Amazon. In: Carolsfeld, J.; Harvey, B.; Ross, C.; Baer, A. (Eds). Biology, fisheries and conservation status. p. 233-301.

Bonilla-Castillo, C.A.; Agudelo; E.; Gómez, G.; Duponchelle, F. 2018. Population dynamics of Prochilodus nigricans (Characiformes: Prochilodontidae) in the Putumayo River.
Neotropical Ichthyology, 16(2): e170139. DOI: http://dx.doi.org/10.1590/1982-022420170139

Catarino, M.F.; Campos, C.P.; Garcez, R.; Freitas, C.E. de C. 2014. Population dynamics of Prochilodus nigricans caught in Manacapuru Lake (Amazon Basin, Brazil). Boletim do Instituto de Pesca, 40(4): 589-295.

Caraballo, P.; Forsberg, B.; Leite, R.G. 2012. Papel trófico del microbial loop en un lago de inundación de Amazonía Central. Acta Biológica Colombiana, 17(1): 103-116.

Castro, R.M.C.; Vari, R.P. 2003. Prochilodontidae (Fannel mouth characiforms). In: Reis, R.E.; Kullander, S.O.; Ferraris Jr. C.J. (Eds). Checklist of the freshwater fishes of South and Central America. Porto Alegre, Brasil. p. 65-70.

Cella-Ribeiro, A.; Hauser, M.; Nogueira, L.D.; Doria, C.R.C.; Torrente-Vilara, G. 2015. Length-weight relationships of fish from Madeira River, Brazilian Amazon, before the construction of hydropower plants. Journal of Applied Ichthyology, 31(5): 939-945. DOI: https://doi.org/10.1111/jai.12819

Chirif, A. 2012. La historia del Tahuayo contada por sus moradores. Wildlife Conservation Society, Lima, Perú. 99pp.

Chuctaya, J.; Capitani, L.; Faustino, D.; Castro, E. 2017. Length-weight relationships of 23 fish species from floodplain ecosystems of the Andean Amazon piedmont, Peru. Journal of Applied Ichthyology, 34(1): 172-176. DOI: https://doi.org/10.1111/jai.13519

Coomes, O.T. 1995. A century of rain forest use in western Amazonia: Lessons for extractionbased conservation of tropical forest resources. Forest \& Conservation History, 39(3):108-120. DOI: https://doi.org/10.2307/3983515

De la Hoz, J.; Duarte, L.O.; Manjarrés-Martínez, L. 2015. Aspectos biológico-pesqueros de especies capturadas en aguas marinas y continentales de Colombia. Relaciones biométricas e indicadores 
basados en tallas. Autoridad Nacional de Acuicultura y Pesca. Bogotá. 36pp.

Dieb-Magalhaes, L.; Florentino, A.C.; Mota Soares, M.G. 2015. Length-weight relationships and length at first maturity for nine fish species of floodplain lakes in Central Amazon (Amazon Basin, Brazil). Journal of Applied Ichthyology, 31(6): 1182-1184. DOI: https:// doi.org/10.1111/jai.12919.

Espinoza, J.C.; Guyot, J.L.; Ronchail, J.; Cochonneau, G.; Filizola, N.; Fraizy, P.; Labat, D.; de Oliveira, E.; Ordoñez, J.J.; Vauchel, P. 2009. Contrasting regional discharge evolutions in the Amazon Basin (1974-2004). Journal of Hydrology, 375(3-4): 297-311. DOI: https://doi.org/10. 016/j.jhydrol.2009.03.004

Famoofo, 0.0.; Abdul, W.O. 2020. Biometry, condition factors and length-weight relationships of sixteen fish species in Iwopin fresh-water ecotype of Lekki Lagoon, Ogun State, Southwest Nigeria. Heliyon, 6(1): e02957. DOI: https://dx.doi.org/10.1016\%2Fj.heliyon. 2019.e02957

Felipa, G.; Blas, W.; Alcántara, F. 2016. Relación longitud-peso, factor de condición y tabla estándar del peso de mil alevinos de gamitana Colossoma macropomum (Cuvier, 1818) criados en estanques. Folia Amazónica, 25(1): 17-24. DOI: https://doi.org/10.24841/fa. v25i1.379

Flores-Gómez, S. 2015. Parámetros reproductivos de Llambina Potamorhina altamazonica (Characiformes: Curitmatidae) en el río Ucayali. Revista de Investigaciones Veterinarias del Perú, 26(2): 223-234. DOI: http://dx.doi. org/10.15381/rivep.v26i2.11004

Froese, R. 2006. Cube law, condition factor and weight-length relationships: history, meta-analysis and recommendations. Journal of Applied Ichthyology, 22(4): 241253. DOI: https://doi.org/10.1111/j.14390426.2006.00805.x
Froese, R.; Pauly, D. 2000. FishBase 2000. Concepts, design and data sources. International Center for Living Aquatic Resources Management (ICLARM), Laguna, Philippines. 344pp.

Froese, R.; Tsikliras, A.C.; Stergiou, K.I. 2011. Editorial note on weight-length relations of fishes. Acta Ichthyologica et Piscatoria, 41(4): 261-263. DOI: https://doi.org/10.3750/ aip2011.41.4.01

Froese, R.; Pauly, D. (Eds.) 2019. FishBase. (https: //www.fishbase.org). Acceso: 01/12/2019.

Frota, L.O.; Costa P.A.S.; Braga, A.C. 2004. Lengthweight relationships of marine fishes from the central Brazilian cost. NAGA, WorldFish Center Quaterly, 27: 20-26.

García, A.; Vargas, G.; Rodríguez, R.; Montreuil, V.; Ismiño, R.; Sanchez, H.; Tello, S.; Duponchelle, F. 2010. Aspectos Biológico Pesqueros de Potamorhina altamazonica llambina (Cope, 1878) en la Región Loreto, Amazonía Peruana. Folia Amazónica, 19(1-2): 23-28. DOI: https:// doi.org/10.24841/fa.v19i1-2.338

García, A.; Tello, S.; Vargas, G.; Duponchelle, F. 2009. Patterns of commercial fish landing in the Loreto Region (Peruvian Amazon) between 1984 and 2006. Fish Physiology and Biochemistry, DOI: https://doi.org/10.1007/ s10695-008-9212-7

García-Ayala, J.R.; Brambilla, E.M.; David, G.S.; Benine, R.C.; Sanchez, H. 2017. Length-weight relationships for seven fish species of the Napo River, Upper Amazon, Peru. Journal of Applied Ichthyology, 1-3. DOI: https://doi. org/10.1111/jai.13428

García-Dávila, C.; Sánchez, H.; Flores, M.; Mejia, J.; Angulo, C.; Castro-Ruiz, D.; Estivals, G.; García, A.; Vargas, G.; Nolorbe, C.; Núñez, J.; Mariac, C.; Duponchelle, F.; Renno, J.F. 2018. Peces de consumo de la Amazonía peuana. Instituto de Investigaciones de la Amazonía Peruana (IIAP). Iquitos, Perú. 218pp. 
Giarrizzo, T.; Bastos D.; Andrade, M. 2011. Length-weight relationships for selected fish species of Rio Trombetas Biological Reserve: a reference study for the Amazonian basin. Journal of Applied Ichthyology, 27(6): 14221424. DOI: https://doi.org/10.1111/j.14390426.2011.01820.x

Goulding, M.; Venticinque, E.; Ribeiro, M.L.de B.; Barthem, R.; Leite, R.L.; Forsberg, B.; Petry, P.; da Silva-Junior, U.L.; Ferraz, P.S.; Cañas, C. 2018. Ecosystem-based management of Amazon Fisheries and wetlands, Fish and Fisheries, 1-21. DOI: $10.1111 /$ faf.12328.1980

Goulding, M. 1980. The fishes and the forest: Explorations in Amazonian natural history. Berkeley \& Los Angeles: University of California Press. 280pp.

Hanjavanit, C.; Buromra, S.; Sangpradub, N. 2013. The length-weight relationships, condition factors and gut contents of Syncrossus helodes (Sauvage, 1876) and Yasuhikotakia modesta (Bleeker, 1864) from the Mekong River, Muang District, Nong Khai Province, Northeastern Thailand. African Journal of Agricultural Research, 8(44): 5508-5517.

Junk, W.; Bayley, P.B.; Sparks, R.E. 1989. The flood pulse concept in rIver-floodplain systems. En: Dodge, D.P. (Ed). Proccedings of the International Large River Symposium. Canadian Special Publication of Fisheries and Aquatic Sciences, 106: 110-127.

Le Cren, E.D. 1951. The length-weight relationship and seasonal cycle in gonad weight and condition of the Pearch (Percha fluviatilis). Journal of Animal Ecology, 20(2): 201-2019.

Lizama, M.A.; Takemoto, R.; Pavanelli, G.C. 2006. Parasitism influence on the hepato, splenoomatic and weight/length relation and relative condition factor of Prochilodus lineatus (Valenciennes, 1836) (Prochilodontidae) of the Upper Paraná River floodplain, Brazil.
Revista Brasileira de Parasitología Veterinaria, 15(3): 116-122.

Marina de Guerra del Perú, Servicio de Hidrografía y Navegación de la Amazonía. 2018. Avisos a los Navegantes Fluviales, edición noviembre 2018.

Mensah, S. 2015. Weight-length models and relative condition factors of nine (9) freshwater fish species from the Yapei stretch of the White Volta, Ghana. Elixir Applied Zoology, 79: 30427 30431.

MINAM, 2019. Sexto informe nacional sobre diversidad biológica. La biodiversidad en cifras. Ministerio del Ambiente, Viceministerio de Desarrollo Estratégico de los Recursos Naturales. Lima, 49 pp.

Morato, T.; Afonsoa, P.; Lourinhoa, P.; Barreiros, J.P.; Santosa, R.S.; Nash, R.D.M. 2001. Lengthweight relationships for 21 coastal fish species of the Azores, north-eastern Atlantic. Fisheries Research, 50(3): 297-302. DOI: https://doi. org/10.1016/S0165-7836(00)00215-0

Mortillaro, J.M.; Abril, G.; Moreira-Turcq, P.; Sobrinho, R.L.; Pérez, M.; Meziane, T. 2011. Fatty acid and stable isotope (d13C, d15N) signatures of particulate organic matter in the lower Amazon River: Seasonal contrasts and connectivity between floodplain lakes and the mainstem. Organic Geochemistry, 42(10): 1159-1168. DOI: http://dx.doi.org/10.1016/j. orggeochem.2011.08.011

Olaya-Nieto, C.; Tordecilla-Prieto, G.; SeguraGuevara, F. 2008. Relación longitud-peso del Rubio (Salminus affinis Steindachner, 1880) en la cuenca del río Sinú, Colombia Revista MVZ Córdoba, 13(2): 1349-1359.

Padoch, C.; Steward, A. 2010. The várzea: The decade past. In: Pinedo-Vasquez, M.; Ruffino, M.L.; Padoch, C.; Brondízio, E.S. (Eds). The Amazon várzea. The decade past and the decade ahead. New York, pp v-xv. DOI: https:// doi.org/10.1007/978-94-007-0146-5 
Prieto, E.F. 2018. Ecología Trófica de un Lago de Varzea (Amazonas, Colombia). Tesis Doctoral. Universidad de Murcia. Facultad de Biología. Murcia, España. 157pp.

Ricker, W.E. 1975. Computation and interpretation of biological statistics of fish populations. Bulletin of the Fisheries Research Board of Canada 191. Ottawa, Canada. 382pp.

Riofrío, J.C. 2002. Aspectos biométricos y reproductivos de Boquichico Prochilodus nigricans Agassiz, 1829 (Pisces: Prochilodontidae) en Ucayali, Perú. Revista Peruana de Biología, 9(2): 111-115. DOI: https://doi. org/10.15381/rpb.v9i2.2529

Riofrío-Quijandría, J.C.; Flores-Gómez, S.; Wasig, J.; Yépez, V. 2017. Reproducción, crecimiento y tasa de explotación de Chiochio (Psectrogaster routiloides) en la cuenca del Río Ucayali entre 2008-2012. Revista de Investigaciones Veterinarias del Perú, 28(2): 345-358. DOI: http://dx.doi.org/10.15381/rivep.v28i2. 13083

Ruffino, M.L.; Isaac, V.J. 1995. Life cycle and biological parameters of several brazilian Amazon fish species. Naga ICLARM Q., 18(4):41-45.

Saint-Paul, U.; Zuanon, J.; Villacorta-Correa, M.A.; García,M.; Fabré,N.N.; Berger,U.; Junk,W.J.2000. Fish communities in central Amazonia white and blackwater floodplains. Environmental Biology of fishes, 57: 235-250. DOI: https:// doi.org/10.1023/A:1007699130333

Sarkar, U.K.; Khan, G.E.; Dabas, A.; Pathak, A.K.; Mir, J.I.; Rebello, S.C.; Pal, A. and Singh, S.P. 2013. Length weight relationship and condition factor of selected freshwater fish species found in River Ganga, Gomti, and Rapti, India. Journal of Environmental Biology, 34: 951-956
Schneider, J.C.; Laarman, P.W.; Gowing, H. 2000. Length-weigth relationships. In: Schneider, James C. (Ed). Manual of fisheries survey methods II: with periodic updates. pp 1-16.

Smith, R.C.; Pinedo, D.; Summers, P.M.; Almeyda, A. 2001. Tropical rhythms and collective action. Community-based fisheries management in the fase of amazonian unpredictability. IDS Bulletin, 32 (4): 36-46. DOI: https://doi. org/10.1111/j.1759-5436.2001.mp32004 005.x

Tello, S.; Bayley, P. 2001. La pesquería comercial de Loreto con énfasis en el análisis de la relación entre la captura y esfuerzo pesquero de la flota comercial de Iquitos, Cuenca del Amazonas (Perú). Folia Amazónica, 12(1-2): 122-139. DOI: https://doi.org/10.24841/fa. v12i1-2.128

Tobes, I.; Miranda, R.; Pino-del-Carpio, A.; AraujoFlores, J.M.; Ortega, H. 2016. Length-weight relationships of freshwater fishes of the Alto Madre de Dios River (Manu Biosphere Reserve, Peru). Journal of Applied Ichthyology, 32: 1256-1258. DOI: https://doi.org/10.1111/jai. 13172

Yossa, M.I.; Araujo-Lima, C.A.R.M. 1998. Detritivory in two Amazonian fish species. Journal of Fish Biology, 526): 1141-1153. DOI: https://doi.org/10.1111/j.1095-8649.1998. tb00961.x

Zargar, U.R.; Yousuf, A.R.; Mushtaq, B.; Dilafroza, J. 2020. Length-weight relationship of the crucian carp, Carassius carassius in relation to water quality, sex and season in some lentic water bodies of Kashmir Himalayas. Turkish Journal of Fisheries and Aquatic Sciences, 12: 683-689.

Recibido: 10 de setiembre de 2020 Aceptado para publicación: 7 de octubre de 2020

Esta obra está bajo una Licencia Creative Commons Atribución-NoComercial-SinDerivar 4.0 Internacional. 